\title{
Progress of Studies on the Fouling of Membrane in Membrane Bioreactor
}

\author{
Jingya Zhang, Huaming Xu \\ Henan University of Traditional Chinese Medicine, Zhengzhou, Henan, P. R. China, 450000
}

Keywords: Membrane bioreactor, Membrane fouling, Membrane fouling control

\begin{abstract}
Progress of studies in the mechanism of the membrane fouling of Membrane Bioreactor was introduced from aspects of membrane characteristics, the operating conditions and the properties of microorganisms the feedwater. The usual control strategies of the membrane fouling were summarized such as optimizing membrane module design, adjusting operating conditions, on-line ultrasound and chemical cleaning. The further research membrane fouling is prospected.
\end{abstract}

\section{Definition of Membrane Fouling}

The definition of membrane fouling given by International Union of Pure and Applied Chemistry (IUPAC) is membrane pollution refers to the suspension or soluble substances deposited on the surface of porous membrane and pore wall, causing the process of membrane flux reduction in membrane fouling process, when the contaminant particles hours than membrane hole, particle adsorption inside the membrane pores, which may lead to the membrane pore blockage. When contaminant particles are bigger than the membrane holes, particles adsorbed on the surface of the membrane will cause the decline of membrane flux.

In terms of the membrane bioreactor, there are 5 following mechanisms which can lead to membrane fouling occurred. (1) the adsorption membrane on the solute and colloidal solution; (2) sedimentation of sludge flock in the membrane surface; (3) the formation of the cake layer on the membrane surface; (4) the separation of pollutant diffusion of shear stress leads to; (5) long-term changes in operation process of pollutants in the composition and properties of the activated sludge in membrane bioreactor traits is complex and changeable. The process of membrane fouling of membrane bioreactor compared with other types of membrane device is more complicated.

\section{Research of Membrane Fouling Mechanism}

Influencing factors of membrane fouling can be divided into three aspects. They are structure properties, membrane reactor operating conditions and the processing liquid microbial properties.

The Structural Natures of Membrane. Structural natures of membrane include membrane material, membrane pore size, hydrophobicity, porosity and surface roughness etc. The structural natures of membrane fouling determines the membrane pollution to a certain extent, may achieve the degree and to restore the membrane fouling degree of difficulty. According to the shape of the commonly used solid membrane, membrane can be divided into the hollow fiber membrane and flat sheet membrane, advantages and characteristics of each are not identical. Anti-pollution more flat membrane can make the system of long-term, stable operation. In the treatment of municipal wastewater, compared with polyethylene membrane of fluoride membrane are more prone to irreversible fouling. Anaerobic membrane bioreactor fluoride membrane than the membrane, cellulose membrane pollution resistance performance is good, the membrane pore size in $0.1 \mathrm{M}$ anti-pollution performance of the best. Shimizu of different aperture membrane bioreactor 
membrane in a series of membranes were studied, the results showed that the pore size distribution in the $0.05-0.20 \mathrm{~m}$ membrane pollution resistance performance of the best. Chang found that the hydrophobic membrane, membrane resistance than hydrophilic membrane, the membrane pollution is more serious. Membrane surface roughness increases not only increase the membrane surface adsorption of contaminants, but also enhances the membrane surface of the disturbance and its effect on membrane fouling.

Reactor Operating Conditions. Reactor operating conditions include the inlet properties, aeration, sludge loading, sludge age, operating temperature and pressure etc. In general, the higher the reactor load is easy to form the membrane fouling, membrane flux decline faster. Aeration is the most important influence of operation conditions of the membrane filtration performance. Effect of fluid turbulence aeration membrane biological reactor and the dynamic degree, can remove the membrane on the surface of the filter cake. But the high strength aerated will make the sludge breakage, may aggravate the membrane pore blocking problem. Membrane bioreactor has a critical operating pressure, when the pressure value is less than the critical operating pressure, membrane flux increases with the increase of pressure increases, when the pressure value is higher than the critical value, the membrane fouling speed, little change in membrane flux. The operation conditions of sludge loading, sludge age and hydraulic retention time can be changed by the activated sludge properties and membrane fouling process of indirect effect.

Processing Liquid Microbial Properties. The main sewage treatment microorganism is a membrane bioreactor, and is also important factor causing membrane pollution. Extracellular Polymers (EPS) and Soluble Microbial Product (SMP) is the impact of the main factors of membrane fouling organisms. EPS is a polymer material producing microbes under certain conditions, generally exists in the activated sludge internal and surface, composition for polysaccharide, protein and DNA. EPS can be divided into two types, one is the cyst polymer attached to the cell wall, the other is a viscous polymer to colloid or dissolved form exists in the processing liquid. EPS can produce a bridging role in microbial cells, the cell aggregation on the membrane surface, cells can also transfer of matter and energy through the EPS, plus EPS play the reproduction of microorganisms and cluster, caused by the blockage of the membrane caused by the decline of membrane flux. So found that polysaccharide membrane pollution in EPS with the closely related Su. Nagaoka research found that the reactor can increase the accumulation of EPS in treated liquid viscosity. The membrane filtration resistance increased. The presence of EPS on sludge sedimentation also affected the main components, such as protein, carbohydrate and DNA content of EPS and sludge volume index (SVI) in proportion to the content is high, the sludge surface negative charge. The sludge EPS effects in repulsion decrease the effect of variation of settlement.

\section{Membrane Used for Controlling Membrane Pollution}

Membrane Pollution can be divided into reversible and irreversible pollution. The pollution which can't be removed by any method is called irreversible pollution. We should take measures to prevent the irreversible pollution, and take measures to remove the reversible pollution. Usually the control of membrane fouling has the following several methods:

Selecting the Suitable Membrane Materials. We can optimize the design of membrane bioreactor and improve the internal structure of the membrane bioreactor. This method can improve the liquid in the reactor flow condition, reduce membrane pollution. In the design, we should pay attention to avoid the water flow dead zone in the reactor appeared. We should be based on the 
nature of sewage treated, choose the suitable membrane materials. Membrane module design should be fully considered placing membrane assembly and hydraulic configuration.

Regulating Membrane Bioreactor Operating Conditions. We should change the pressure mode membrane biological reactor, using intermittent suction or pressure increasing pattern. It can slow the membrane fouling. Usually the membrane bioreactor in the early operation can be run at low pressure. With the aggravation of pollution, it need to improve the membrane filtration pressure to stabilize the membrane flux, operating pressure should be smaller than the critical operating pressure value.

Water back flushing or air reverse blowing can be membrane surface cake layer stripping, cleaning out the pollutants in the membrane hole. The backwashing membrane is generally carried out in fixed cycle. To avoid membrane pollution to the critical membrane pollution level, because the membrane cleaning removal of membrane pollution has been difficult to play a role. Given to the pressure the membrane can withstand, we should use the reverse washing method to avoid the influence on membrane structure function.

Online Ultrasonic Control. The online ultrasonic cleaning device is arranged in the membrane bioreactor. Mechanical vibration generated in the water by use of ultrasonic wave and micro turbulence effect can make the pollutants detach from the membrane surface. Online ultrasonic cleaning is a control method of membrane pollution is relatively new, the utility mode. It has the advantages of obvious effect, convenient operation and no damage to the membrane itself.

Using Chemical Method. Chemical cleaning is a common method to control membrane fouling. The use of alkaline cleaning agent, can remove the membrane protein and other organic pollutants, can damage the cake layer, make it fall off from the membrane surface, commonly used with sodium hydroxide, potassium hydroxide. The use of acidic cleaning agent can accumulate dissolve and remove membrane minerals, commonly used with nitric acid, citric acid, such as hydrochloric acid. The mixed solution into the membrane bioreactor often contains various impurities can be by adjusting the $\mathrm{pH}$ value and adding antioxidants, adding anti scaling agent and flocculants on the pretreatment, to reduce membrane pollution. Shona tested 4 different pretreatment methods and found that plus adsorption method can effectively prevent membrane fouling. Chemical cleaning only single pollutant reduction viscosity on the film cannot be directly remove pollutants. The chemical cleaning and water cleaning, ultrasonic cleaning combined can significantly improve the removal effect on membrane fouling.

\section{Conclusions and Suggestions}

We should carry out some methods to control membrane fouling at the same time. In the selection of membrane bioreactor, should according to the properties required for sewage treatment, choose the suitable membrane materials. The membrane biological reactor is in operation, can be adjusted by the hydraulic operating conditions and the use of chemical methods to control membrane pollution. To develop membrane materials and development of anti-pollution ability is an important way to control membrane fouling.

At present, people's understanding of the mechanism of membrane fouling is not comprehensive. Many aspects of membrane fouling process still need further study. The growing demand of sewage treatment technology and equipment and the need of the sewage treatment cost control is the impetus of membrane pollution of the membrane fouling problem. With membrane bioreactor gradually resolved and the invention of economic efficient membrane material, the membrane bioreactor will be used more widely. 


\section{References}

[1] F.G. Meng, So-Ryong Chae, Anja Drews, et al.Recent advances in membrane bioreactors (MBRs):Membrane fouling and membrane material, Water Res., 2009, 43(6): 1 489- 1512.

[2] S Delgado, R Villarroel, E Gonz lez. Effect of the shear intensity on fouling in submerged membrane bioreactor for wastewater treatment, Journal of Membrane Science, 2013, 311(1- 2): 173- 181.

[3] $\mathrm{N}$ Yamato, K Kimura, $\mathrm{T}$ Miyoshi, et al. Difference in membrane fouling in membrane bioreactors (MBRs) caused by membrane polymer materials. Journal of Membrane Science, 2012, 280(1- 2): 911- 919.

[4] Choo K H, Lee C H. Effect of anaerobic digestion broth composition on membrane permeability [J]. Water Sci. Tech., 2012, 34(9): 173- 179. 